\section{Hightech-Metalle für Zukunftstechnologien}

\author{
von Gerhard Angerer, Karlsruhe
}

Die Sicherung der Rohstoffversorgung ist eine Herausforderung für die Zukunft, deren Bedeutung mit der des Klimaschutzes vergleichbar ist. Es wäre fatal, wenn die Entwicklung, Nutzung und Vermarktung von Zukunftstechnologien durch Engpässe bei der Versorgung mit Rohstoffen erschwert oder gar verhindert würden. Die Fragestellungen der Studie, über die hier berichtet wird, sind, welche Impulse von der späteren Nutzung der heute oft noch im Entwicklungsstadium befindlichen Zukunftstechnologien auf die Rohstoffnachfrage ausgehen und auf welche Rohstoffe diese Zukunftsinnovationen besonders angewiesen sind. Am Beispiel ausgewählter Zukunftstechnologien wird gezeigt, zu welchen Ergebnissen ein Foresight des Bedarfs nach Hightech-Metallen kommt.

\section{Turbulente Rohstoffmärkte}

Die Industriestaaten gewinnen als Hochlohnländer im globalen Markt durch technische Innovationen Wettbewerbsvorteile. Der durch diese Tatsache ausgelöste Forschungs- und Entwicklungs- wettlauf erhöht die Innovationsgeschwindigkeit fortwährend. Zugleich ist die deutsche Wirtschaft nicht nur bei Energierohstoffen, sondern auch bei Metallen beinahe vollkommen von Importen abhängig. Der Erfolg Deutschlands im Export seiner Produkte der Hoch- und Spitzentechnologie, und damit der Wohlstand der Gesellschaft, sind deshalb auf eine störungsfreie Versorgung mit Rohstoffen zu angemessenen Preisen angewiesen.

Die Lage auf den Rohstoffmärkten war in den zurückliegenden Jahren hoch turbulent. Neue Marktteilnehmer aus den Schwellenländern, allen voran China, haben unerwartet starke Impulse auf die Rohstoffnachfrage ausgelöst. Die Nachfrageeffekte technischer Innovationen wurden nicht rechtzeitig erkannt und führten zu Fehleinschätzungen auf den Rohstoffmärkten. Dies ließ die Rohstoffpreise sprunghaft steigen und hat die Materialkosten im deutschen Verarbeitenden Gewerbe nach oben getrieben, wie Abbildung 1 zeigt. Ein Ende der Turbulenzen ist nicht in Sicht. Mit der globalen Krise des Bankensystems sind die Rohstoffpreise kurzzeitig stark gefallen, bewegen sich aber schon wieder steil nach oben.

Veränderungen der Rohstoffpreise treffen mit den Materialkosten den mit Abstand größten Kostenblock in der Produktion. Tabelle 1 gibt die vom Statistischen Bundesamt ermittelten Antei-

Abb. 1: Preisbereinigte Kostenentwicklung im Verarbeitenden Gewerbe in Deutschland

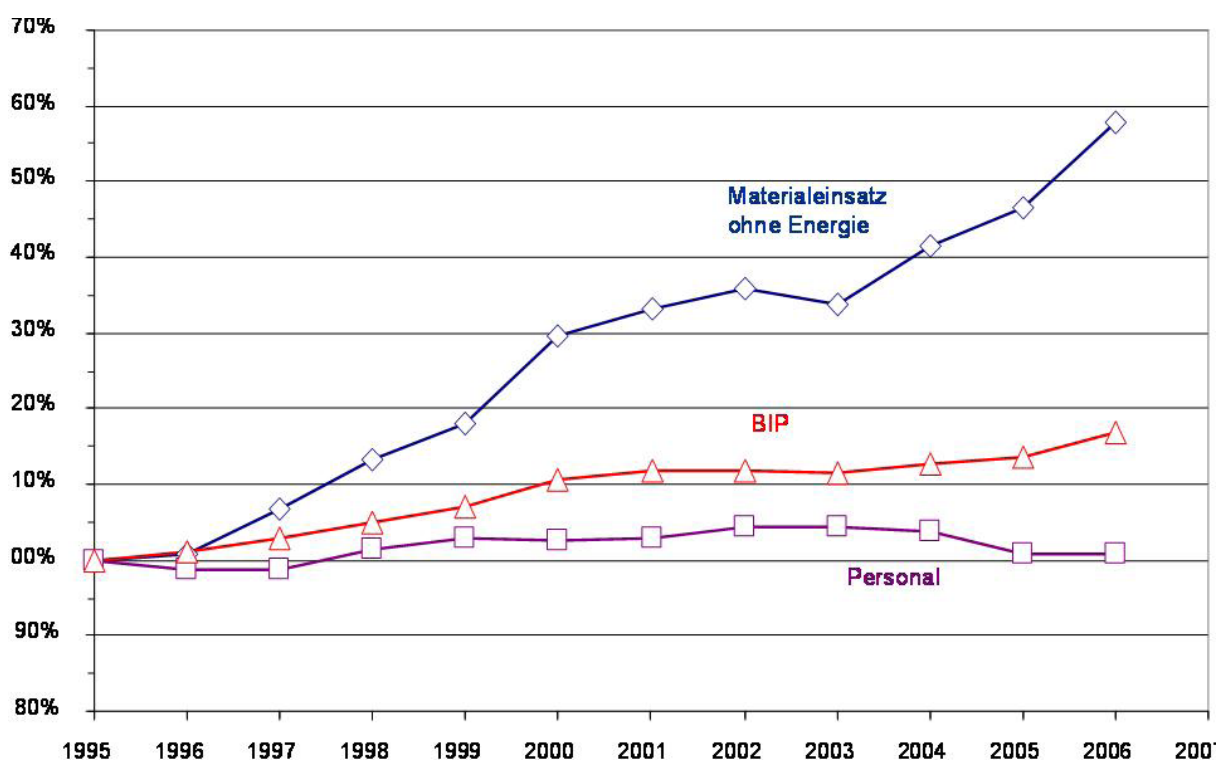

Quelle: Stat. Bundesamt, Fachreihe Produzierendes Gewerbe, versch. Jahrgänge; Stat. Bundesamt, Fachreihe Volkswirtschaftliche Gesamtrechnung 2007; Stat. Bundesamt 2007 
le am Bruttoproduktionswert des Verarbeitenden Gewerbes für das Jahr 2006 wieder.

Tab. 1: Kostenstruktur im Verarbeitenden Gewerbe Deutschlands (2006)

\begin{tabular}{lc}
\hline Kostenart & Anteil in \% \\
\hline Materialkosten & 43,0 \\
Energiekosten & 1,8 \\
$\begin{array}{l}\text { Personalkosten, Lohnarbeiten und hand- } \\
\text { werkliche Dienstleistungen }\end{array}$ & 22,7 \\
$\begin{array}{l}\text { Übrige Kosten (Einsatz von Handelsware, } \\
\text { Steuern, Abschreibungen etc.) }\end{array}$ & 32,5 \\
\hline Bruttoproduktionswert ohne Umsatzsteuer & 100,0 \\
\hline Quelle: Stat. Bundesamt, Fachreihe Produzierendes
\end{tabular}

Aber es sind nicht nur die Produktionskosten und Produktpreise, welche durch die Rohstoffe getrieben werden, der Rohstoffabbau, ihre Verhüttung und Weiterverarbeitung zu Werkstoffen und Vorprodukten ist auch mit Umweltlasten verbunden. Der Abbau seltener Rohstoffe, wie beispielsweise Indium, lässt ihre Ressourcen merklich schwinden und konfligiert mit dem Leitbild einer nachhaltigen Entwicklung.

Vor diesem Hintergrund sind die Sicherung der Rohstoffzugänge und der sparsame Umgang mit Ressourcen sowohl unter Nachhaltigkeits- als auch unter Kosten- und Wettbewerbsgesichtspunkten eine Zukunftsaufgabe, deren Bedeutung der des Klimaschutzes gleich kommt. Dieser Einsicht ist es zu verdanken, dass diese Herausforderung in Politik, Wirtschaft und Wissenschaft mehr und mehr Aufmerksamkeit gewinnt (Reuscher et al. 2008; Rohn et al. 2009; vbw 2009; Ziemann 2009; Teipel 2010). Angespornt durch die Energieforschung und -entwicklung, die sich seit der ersten Ölkrise 1973 ohne Unterbrechung um die Verbesserung der Energieeffizienz bemühen, und dabei nicht für möglich gehaltene Erfolge erzielt haben, werden auch in der Materialwirtschaft epochale Fortschritte für möglich gehalten.

Die Fragestellungen der Studie, deren Ergebnisse hier vorgestellt werden, waren, welche Impulse von der späteren Nutzung der heute oft noch im Entwicklungs- oder Pilotstadium befindlichen Zukunftstechnologien auf die Rohstoffnachfrage ausgehen und auf welche Rohstoffe diese Innovationen besonders angewiesen sind.
Die Ergebnisse wurden unter der Leitung des Fraunhofer-Instituts für System- und Innovationsforschung (ISI) zusammen mit dem Institut für Zukunftsstudien und Technologiebewertung (IZT) im Auftrag des Bundesministeriums für Wirtschaft und Technologie in der Zeit von Januar bis November 2008 erarbeitet (Angerer et al. 2009). Anlass des Auftrags waren die seit 2003 zu beobachtenden exorbitanten Steigerungen der Rohstoffpreise, welche die Sorge um mögliche Versorgungsengpässe nährte.

Zukunftstechnologien sind industriell verwertbare technische Fähigkeiten, die revolutionäre Innovationsschübe weit über die Grenzen einzelner Wirtschaftssektoren hinaus auslösen und langfristig tiefgreifend die Wirtschaftsstrukturen, das Sozialleben und die Umwelt verändern. Innovationsschübe können Einzeltechniken betreffen, wie beispielsweise Brennstoffzellen, organische Leuchtdioden oder RFID-Labels. Es können aber auch „systemische Innovationsschübe“ sein, die bekannte Einzeltechniken zu neuen Anwendungen verbinden. Beispiele sind das Hybridauto oder die thermochemische Produktion von synthetischen Kraftstoffen aus Biomasse. Es ist unschwer erkennbar und wurde schon eingangs erwähnt, dass der Beherrschung und Vermarktung von Zukunftstechnologien für die deutsche Wirtschaft und deren Wettbewerbsstellung in der Welt essenzielle Bedeutung zukommt.

\section{Der Blick in die Zukunft: Foresight}

Zukunftstechnologien lassen sich nicht auf fünf, zehn oder 20 Innovationen eingrenzen. Vielmehr ist in allen Sektoren eine grundlegende Erneuerung der Volkswirtschaft im Gange, die von dem Ziel der industrialisierten Hochlohnländer getrieben wird, im globalen Wettbewerb durch technologische Exzellenz zu bestehen. Für die Untersuchung war es daher nötig, die Zahl der analysierten Technologien und der einbezogenen Rohstoffe auf ein bearbeitbares $\mathrm{Ma} ß \mathrm{zu}$ beschränken. Die Auswahl der Rohstoffe orientierte sich an der Einschätzung ihrer Bedeutung für die Technologieentwicklung. Weil Energierohstoffe seit der ersten Ölkrise 1973 Gegenstand von Analysen sind, beschränkte sich das vorliegende Projekt auf anorganische, nicht energetisch ge- 
nutzte mineralische Rohstoffe. Weil Deutschland bei den Metallen fast vollständig von Importen abhängig ist, wurden ausschließlich metallische und halbmetallische Rohstoffe einbezogen.

Potenzielle Versorgungsrisiken gehen von „vulnerablen Rohstoffen“ aus. Vulnerabel sind Rohstoffe, wenn sie hohe Bedeutung für die Volkswirtschaft haben, ihre Vorkommen auf wenige Länder konzentriert sind und diese in einer politisch instabilen Region liegen (Frondel et al. 2007). Weil es in erster Linie solche Rohstoffe sind, welche die Entwicklung und industrielle Nutzung von Zukunftstechnologien hemmen könnten, stehen sie im Fokus der Aufmerksamkeit. Weitere Auswahlkriterien waren ihre vermutete funktionelle und mengenmäßige Bedeu- tung für die Entwicklung und spätere Nutzung von Zukunftstechnologien. Das Rohstoffportfolio der einbezogenen Rohstoffe ist in Tabelle 2 zusammengestellt.

\section{Tab. 2: Das Rohstoffportfolio}

\begin{tabular}{ll}
\hline „Commodities”* & Spezialitäten \\
\hline 1. Antimon & 9. Platinmetalle - Pt, Pd, Ru, \\
2. Chrom & Rh, Os, Ir \\
3. Kobalt & 10.Silber \\
4. Kupfer & 11.Seltene Erden - Sc, Y, Nd \\
5. Niob & 12.Selen \\
6. Tantal & 13.Indium \\
7. Titan & 14.Germanium \\
8. Zinn & 15.Gallium
\end{tabular}

* = Commodities sind Massenrohstoffe mit breitem Verwendungsspektrum.

Quelle: Eigene Darstellung

Tab. 3: Das Portfolio analysierter Zukunftstechnologien

\begin{tabular}{|c|c|}
\hline Fahrzeugbau, Luft- und Raumfahrt, Verkehrstechnik & $\begin{array}{l}\text { Stahlleichtbau mit Tailored Blanks } \\
\text { Elektrische Traktionsmotoren für Kraftfahrzeuge } \\
\text { Brennstoffzellen Elektrofahrzeuge } \\
\text { Superkondensatoren für Kraftfahrzeuge } \\
\text { Scandium-Legierungen für den Airframe-Leichtbau }\end{array}$ \\
\hline Informations- und Kommunikationstechnik, optische & Bleifreie Lote \\
\hline Technologien, Mikrotechniken & $\begin{array}{l}\text { RFID - Radio Frequency Identification } \\
\text { Indium-Zinn-Oxid (ITO) in der Displaytechnik } \\
\text { Infrarot-Detektoren in Nachtsichtgeräten } \\
\text { Weiße LED } \\
\text { Glasfaserkabel } \\
\text { Mikroelektronische Kondensatoren } \\
\text { Hochleistungs-Mikrochips }\end{array}$ \\
\hline Energie-, Elektro- und Antriebstechnik & $\begin{array}{l}\text { Ultraeffiziente industrielle Elektromotoren } \\
\text { Thermoelektrische Generatoren } \\
\text { Farbstoffsolarzellen } \\
\text { Dünnschicht-Photovoltaik } \\
\text { Solarthermische Kraftwerke } \\
\text { Stationäre Brennstoffzellen - SOFC } \\
\text { CCS - Carbon Capture and Storage } \\
\text { Lithium-Ionen-Hochleistungs-Elektrizitätsspeicher } \\
\text { Redox-Flow-Elektrizitätsspeicher } \\
\text { Vakuumisolation }\end{array}$ \\
\hline $\begin{array}{l}\text { Chemie-, Prozess-, Fertigungs- und Umwelttechnik, } \\
\text { Maschinenbau }\end{array}$ & $\begin{array}{l}\text { Synthetische Kraftstoffe } \\
\text { Meerwasserentsalzung } \\
\text { Festkörper-Laser für die industrielle Fertigung } \\
\text { Nanosilber }\end{array}$ \\
\hline Medizintechnik & $\begin{array}{l}\text { Orthopädische Implantate } \\
\text { Medizinische Tomographie }\end{array}$ \\
\hline Werkstofftechnik & $\begin{array}{l}\text { Superlegierungen } \\
\text { Hochtemperatursupraleiter in der Elektrizitätswirtschaft } \\
\text { Hochleistungs-Permanentmagnete }\end{array}$ \\
\hline
\end{tabular}

Quelle: Eigene Darstellung 
In Tabelle 3 sind die 32 Zukunftstechnologien aufgelistet, die für eine vertiefende Analyse ausgewählt wurden. Nicht rohstoffrelevante Innovationen, beispielsweise Tissue Engineering zur Züchtung von Hautlappen, Herzklappen, Nieren und anderen Organersatzteilen, sind mangels Bedarf an mineralischen Rohstoffen nicht enthalten, obwohl es sich dabei ohne Zweifel um eine Zukunftstechnologie handelt. Das gleiche gilt für innovative Browsertechnologien des Wissensmanagements.

Als Foresightmethode wurde ein Bottomup-Ansatz gewählt. Das heißt, aus einer technisch-wirtschaftlichen Innovationsanalyse wurden die spezifischen Eigenschaften der Technologie, ihr Fortschritt gegenüber dem Stand der Technik, die spezifischen Funktionen, welche die verwendeten Roh- und Werkstoffe zur Verfügung stellen, das mögliche Anwendungsspektrum und die potenziellen Märkte erschlossen. Aus dem gewonnenen Verständnis von Technologie und Markt wurden unter Berücksichtigung der Rahmenbedingungen (darunter die Weltwirtschaftsentwicklung), Projektionen der künftigen Technologienutzung und des daraus resultierenden Rohstoffbedarfs hergeleitet. Zeithorizont des Foresights ist das Jahr 2030, Basisjahr ist 2006.

Zukunftsprojektionen zeigen mögliche Entwicklungen in die Zukunft auf. Sie stützen sich auf bestimmte und explizit zu nennende Annahmen und treten ein, wenn die tatsächliche Entwicklung diesen Annahmen folgt. Die Szenariotechnik gestattet es, von unterschiedlichen Zukunftsentwürfen auszugehen, um so die tatsächlich eintretende Entwicklung in einem Fächer von Projektionen einzufangen. Zukunftsprojektionen dürfen nicht als Vorhersagen der tatsächlichen künftigen Entwicklung verstanden werden. Niemand kann die Zukunft im Jahr 2030 prognostizieren.

Worin liegt dann der Wert von Projektionen? Sie lassen erkennen, welche Einflüsse die künftige Entwicklung treiben und zeigen den Marktteilnehmern, welche Treiber sie beobachten müssen. Marktteilnehmer sind zum einen die Rohstoffkonzerne, welche Informationen über ihre Märkte für die strategische Kapazitätsplanung benötigen. Zum anderen sind es die Rohstoffe verarbeitenden Industrien, die frühzeitig auf Nachfragekonkurrenzen zu anderen Sektoren, potenzielle Roh- stoffknappheiten und sich abzeichnende Substitutionserfordernisse hingewiesen werden.

\section{Der Treiber „,Technischer Wandel“}

Die seit Beginn dieses Jahrzehnts verstärkt zu beobachtenden Turbulenzen auf den Rohstoffmärkten haben ihre Ursache nicht, wie oft irrtümlich angenommen wird, in der Erschöpfung von Rohstoffvorkommen. Die statische Ressourcenreichweite von Chrom beträgt 600 Jahre, von Titan 280 Jahre, von Kobalt 220 Jahre, der Platinmetalle 190 Jahre (USGS 2008), und trotzdem sind die Preise dieser Metalle hoch volatil. Die Marktturbulenzen entstanden aus einem Ungleichgewicht von Angebot und Nachfrage. Sie wurden und werden durch renditeorientierte Finanzgeschäfte verstärkt, die neben der Realwirtschaft getätigt werden. Die Fehleinschätzung der Märkte geht zum einen auf die stürmische Entwicklung der chinesischen Wirtschaft und dem davon ausgelösten Boom der Rohstoffnachfrage zurück, der für viele Marktteilnehmer unerwartet kam. Zum anderen resultiert die Fehleinschätzung aus nicht rechtzeitig antizipierten technischen Entwicklungen. Ein Beispiel ist der Umstieg der Elektro- und Elektronikindustrie auf bleifreie Lote, die einen höhern Zinngehalt aufweisen. Dies hat die Zinnnachfrage und die Zinnpreise stark anziehen lassen.

Die Analyse der von der Nutzung neuer Technologien ausgehenden Impulse auf die Rohstoffnachfrage lässt deutlich die gestaltende Kraft des technischen Wandels erkennen. Dies zeigt Tabelle 4. Sie gibt rohstoffspezifisch das Verhältnis des Rohstoffbedarfs der analysierten $\mathrm{Zu}$ kunftstechnologien zur heutigen gesamten Weltproduktion des jeweiligen Rohstoffs wieder, die in der letzten Spalte angegeben wird. Die Zahlen für 2006 zeigen, welcher Anteil der jeweiligen Weltrohstoffproduktion durch die analysierten Technologien erfasst wird. Die Zahlen für 2030 zeigen, welches Vielfache beziehungsweise welcher Anteil der heutigen Weltproduktion des jeweiligen Rohstoffs für diese Technologien 2030 benötigt werden wird. Sie sind ein Indikator für den Ausbaubedarf der Minenproduktion. Der Indikator erreicht bei Gallium den Faktor 6,09 und bei Neodym 3,8. D. h., der von absehbaren technischen Innovationen ausgehende Bedarf nach 
Gallium wird $20306,09 \times 99$ t/a $=603$ t/a und für Neodym 3,8 x $7.300 \mathrm{t} / \mathrm{a}=27.740 \mathrm{t} / \mathrm{a}$ betragen und damit 2030 weit über der gesamten heutigen Jahresproduktion liegen. ${ }^{1}$

Bedarfstreiber bei Gallium sind die Dünnschicht-Photovoltaik, schnelle integrierte Schaltungen und weiße Leuchtdioden (WLED). Bedarfstreiber bei Neodym sind Hochleistungs-Permanentmagnete. Der Indikator erreicht bei Indium den Faktor 3,3, bei Germanium 2,4, bei Scandium 2,3, bei Platin 1,6 und bei Tantal 1. Für Silber und Zinn beträgt er immerhin noch jeweils 0,8 , bei Kobalt 0,4 und bei Palladium und Titan jeweils 0,3 und bei Kupfer gut 0,2 . Aufgrund des hier erkennbaren dominanten Einflusses des technischen Wandels auf die Rohstoffnachfrage sind dies zugleich jene Rohstoffe des Projektportfolios, die für die künftige Technologieentwicklung und ihre Nutzung in marktfähigen Produkten besonders wichtig sind.

\section{Der Treiber „Weltwirtschaft“}

Vor allem China hat das Wachstum der Weltwirtschaft von einem moderaten Durchschnitt von 3,8 Prozent pro Jahr in den zurückliegenden 20 Jahren auf fünf Prozent pro Jahr zwischen 2004 und 2007 hochschnellen lassen. Wenn es gelingen soll, Angebot und Nachfrage im Gleichgewicht zu halten, dann muss als zweiter Faktor neben dem technischen Fortschritt auch die Entwicklung der Weltwirtschaft im Auge behalten und antizipiert werden. Das zurzeit herrschende Wohlstandsgefälle zwischen Regionen der Welt ist nicht zukunftsfähig. Beispielsweise trägt Afrika mit einer Milliarde Menschen kaum 1,6 Prozent zum globalen Inlandsprodukt bei. Es ist keine konfliktfreie Zukunft vorstellbar, in der die entwickelten Regionen ihren Wohlstand stetig mehren, während die armen Regionen auf ihrem Niveau verharren. Das wirtschaftliche Aufholen der weniger entwickelten Regionen ist Voraussetzung für eine friedliche Zukunft und liegt im Interesse aller Nationen der Welt. Der Ausgleich des Wohlstandsgefälles in einer angemessenen Zeitspanne wird zwangsläufig das Weltwirtschaftswachstum über das langfristige Mittel der Vergangenheit steigen lassen. Aber selbst, wenn nur eine künftige jährliche Wachstumsrate von 3,8 Prozent zugrunde gelegt wird, erreicht die Weltwirtschaftsleistung im Jahre 2030 das 2,4-fache von 2006. Von diesem Wachstum gehen starke Impulse auf den zukünftigen Bedarf an Rohstoffen aus.

Tab. 4: Globaler Rohstoffbedarf des Zukunftstechnologien-Portfolios im Jahr 2006 und $2030 \mathrm{im}$ Verhältnis zur gesamten heutigen Weltproduktionsmenge des jeweiligen Rohstoffs

\begin{tabular}{|c|c|c|c|c|}
\hline \multirow[t]{2}{*}{ Rohstoff } & \multicolumn{2}{|c|}{ Rohstoffbedarf } & \multirow[t]{2}{*}{ Zukunftstechnologien } & \multirow{2}{*}{$\frac{\text { Weltproduktion }}{2006(\text { in } t)^{2}}$} \\
\hline & 2006 & 2030 & & \\
\hline Gallium & 0,28 & 6,09 & Dünnschicht-Photovoltaik, IC, WLED & 99 \\
\hline Indium & 0,40 & 3,29 & Displays, Dünnschicht-Photovoltaik & 580 \\
\hline Germanium & 0,31 & 2,44 & Glasfaserkabel, IR optische Technologien & 90 \\
\hline Scandium & gering & 2,28 & SOFC Brennstoffzellen, Al-Legierungselement & 1,3 \\
\hline Tantal & 0,39 & 1,01 & Mikrokondensatoren, Medizintechnik & 1.400 \\
\hline Silber & 0,26 & 0,78 & RFID, Bleifreie Weichlote & 20.200 \\
\hline Zinn & 0,62 & 0,77 & Bleifreie Weichlote, transparente Elektroden & 302.000 \\
\hline Kobalt & 0,19 & 0,40 & Lithium-Ionen-Akku, XtL & 67.500 \\
\hline Palladium & 0,10 & 0,34 & Katalyse, Meerwasserentsalzung & 224 \\
\hline Niob & 0,01 & 0,03 & Mikrokondensatoren, Ferrolegierung & 45.000 \\
\hline Ruthenium & 0 & 0,03 & Farbstoffsolarzellen, Ti-Legierungselement & 29 \\
\hline Yttrium & gering & 0,01 & Hochtemperatursupraleitung, Lasertechnik & 7.000 \\
\hline Antimon & gering & gering & ATO, Mikrokondensatoren & 134.000 \\
\hline Chrom & gering & gering & Meerwasserentsalzung, marine Techniken & 9.010 .000 \\
\hline
\end{tabular}

Quelle: Angerer et al. 2009 
Welches der dominierende Treiber der Nachfrage ist, lässt sich nicht generell beantworten, sondern hängt vom spezifischen Rohstoff ab. Commodities, unter denen in großen Mengen hergestellte Massenrohstoffe mit breitem Verwendungsspektrum (wie Eisen, Stahl, Kupfer und Chrom) zu verstehen sind, werden eher vom Weltwirtschaftswachstum getrieben. Für Hightech-Spezialitäten wie Gallium, Neodym, Indium, Germanium und Scandium ist eher die technologische Entwicklung bedarfsbestimmend.

Ist angesichts der absehbaren Nachfragesteigerung überhaupt eine sichere Rohstoffversorgung vorstellbar? Grundsätzlich schon. Je nach Komplexität der Technologie vergehen fünf, zehn und mehr Jahre, bis eine neue Technik aus dem Forschungs- und Entwicklungsstadium in die breite Anwendung gelangt. Toyota hat als Pionier in der Hybridtechnologie des Fahrzeugbaus einen Entwicklungsvorsprung von mindestens fünf Jahren. Dies, obwohl die Herausforderung hier ,nur“ darin besteht, bekannte Komponenten, nämlich Verbrennungsmotor und Elektromotor, zu einem System zu integrieren. Die Entwicklungsanstrengungen der Industrie, Brennstoffzellen praktisch nutzbar zu machen, lassen sich bis in die 1960er Jahre zurückverfolgen. Die Beherrschung der Energiegewinnung aus der Kernfusion und die Bereitstellung eines funktionierenden Fusionskraftwerks dürfte nahezu ein Jahrhundert benötigen. Die Zeitspanne für die Umsetzung von Bergbauprojekten, in der Branche als „Lead Time" bezeichnet, beträgt fünf bis zehn Jahre. Den Rohstoffkonzernen steht also ausreichend Anpassungszeit zur Verfügung, dem technischen Fortschritt zu folgen. Technische Entwicklungen rechtzeitig zu erkennen und ihre Richtung und Stärke sicher einzuschätzen, ist allerdings nicht trivial. Hier kann ein regelmäßiger Informationsaustausch zwischen den Rohstoffkonzernen und den Rohstoffe verarbeitenden Industrien helfen, realistische Zukunftsbilder zu entwerfen.

Auf den Rohstoffmärkten agieren nicht nur die Marktteilnehmer der Realwirtschaft, die Märkte sind auch Ziel von renditeorientierten, spekulativen Finanzgeschäften. Welche Rolle letztere bei der Preisbildung spielen, ist offen. Hier besteht (wie bei den Energierohstoffen) Analyse- und wahrscheinlich auch Regulierungsbe- darf. Dominante, preistreibende Spekulationsgeschäfte würden einen gesellschaftspolitisch unerwünschten Wohlstandstransfer herbeiführen, weil die Konsumenten mehr für Rohstoffe aufwenden müssen, als ihrem realen Gegenwert entspricht.

\section{Die Vulnerabilität des Verarbeitenden Gewerbes}

Die Rohstoffversorgung muss als vulnerables System verstanden werden. Sie ist global hoch vernetzt und dadurch vielfältigen Einflüssen ausgesetzt, die von einzelstaatlichen Politiken nur beschränkt gesteuert werden können. Dabei dürften Marktstörungen weniger in einem Stopp der Rohstoffzufuhr zu sehen sein, sondern eher in Lieferverzögerungen, in Konzentrationsbewegungen globaler Konzerne, die Marktmacht erzeugen und den Wettbewerb aushebeln, oder in der Instabilität von Förderländern, die schlagartig die Minenproduktion lahm legen kann, weil der gesellschaftliche Konsens hoch fragil ist (Coltan $\mathrm{Krise}^{3}$, südafrikanisches Chrom). Marktstörungen entstehen auch, wenn unvorhergesehene Prosperität in Schwellen- und Entwicklungsländern die Nachfrage puscht (China, Indien, Brasilien) oder revolutionäre Technologieschübe Bedarfsspitzen (Elektromobilität) oder -einbrüche verursachen.

Die Empfindlichkeit der Rohstoffe verbrauchenden Wirtschaftssektoren ist dort besonderes groß, wo die Möglichkeit fehlt, knappe und teure Rohstoffe zu substituieren. Nicht substituierbar ist beispielsweise Chrom in rostfreien Stählen, Zinn in Weichloten, Kobalt in verschleißfesten Legierungen, Scandium in schlagfesten Aluminium-Scandium-Legierungen, Silber in gedruckten RFID-Labels, Indium in transparenten IndiumZinn-Oxid-Elektroden für Displays, Neodym in starken Permanentmagneten und Germanium in Linsen der Infrarotoptik. Zwischen den Wirtschaftssektoren bestehen zugleich Nachfragekonkurrenzen um Rohstoffe. So wird Tantal für die Herstellung miniaturisierter Kondensatoren in elektronischen Schaltungen, als Ferrolegierung für die Stahlveredelung und als hoch biokompatibles Metall für Stifte, Drähte und Schrauben in der Chirurgie benötigt. Platin ist in der Katalyse der Chemietechnik, in Abgaskatalysatoren für Kraftfahrzeuge, in Brennstoffzellen und in vielen 
anderen Anwendungen unverzichtbar. Silber wird in nanoskaliger Form als Biozid genutzt, ist Bestandteil von Weichloten und wird für hochwertige Verspiegelungen eingesetzt (beispielsweise bei hocheffizienten solarthermischen Rinnenkollektoren). Kobalt bedient zum einen die vielfältigen und stark wachsenden Anwendungen der Lithium-Ionen-Technik, wird als Katalysator in der Fischer-Tropsch-Synthese genutzt (z. B. bei der Kraftstoffsynthese) und ist essenzieller Bestandteil hoch verschleißfester Bauteile im Fahrzeugund Maschinenbau sowie in der Medizintechnik.

\section{Fazit}

Verbundwerkstoffe und komplexe Werkstoffverbünde gewinnen mehr und mehr an Bedeutung - sei es, um die Leistungsfähigkeit von Bauteilen zu steigern (Milchverbundkarton), Gewicht einzusparen (,tailored blanks" ${ }^{\text {(4) }}$, sie zu miniaturisieren (integrierte Schaltkreise), spezifische Stoffeigenschaften einzustellen (Hybridpolymere, ORMOCER ${ }^{\circledR}$ ) oder aus anderen Gründen. Die Trennung hoch komplexer Materialverbünde, wie sie beispielsweise in integrierten Schaltkreisen realisiert sind, und die Rückgewinnung der enthaltenen Wertstoffe stellt höchste Anforderungen an die Recyclingtechnik, die gegenwärtig aus technischen oder wirtschaftlichen Gründen oft noch nicht erreicht werden. Hier gilt es, leistungsfähige Recyclingtechniken bereit zu stellen, die kosteneffizient Sekundärrohstoffe in der Qualität von Primärrohstoffen gewinnen. Sekundärrohstoffe sind bei den Metallen die einzige nennenswerte heimische Rohstoffquelle Deutschlands.

Niemand kann die künftige Entwicklung der Rohstoffmärkte prognostizieren. Die eingehende Analyse ihrer Treiber und die Herausarbeitung der Trends von Angebot und Nachfrage erlauben es jedoch, mögliche zukünftige Entwicklungen einzugrenzen und sich vorsorglich auf sie einzustellen. Die in der zitierten Untersuchung erarbeiteten Trendprojektionen zeigen deutlich in die Richtung einer insgesamt steigenden Rohstoffnachfrage.

Den Unternehmen wird empfohlen, die technologische und weltwirtschaftliche Entwicklung laufend zu beobachten und vorsorglich Maßnahmen zur Rohstoffsicherung zu ergreifen.
Deutschland ist mit seiner Abhängigkeit von importierten Rohstoffen nicht in einer komfortablen Position (GDCh, Dechema, DGMK, VCI 2010). Zudem sind auf der Angebotsseite Konzentrationsbewegungen $\mathrm{zu}$ beobachten, welche die Marktkräfte auszuhebeln drohen. Über 95 Prozent der Weltproduktion an Metallen der Seltenen Erden (darunter Lanthan, Yttrium, Cer, Neodym und Scandium), 60 Prozent des Indiums und 30 Prozent des Galliums stammen aus China. 70 Prozent des Zinns, ein unverzichtbarer Rohstoff für Weichlote, beispielsweise in der Elektronikfertigung, werden in Asien produziert, 56 Prozent des Chroms kommt aus Kasachstan und Südafrika und 55 Prozent des Kobalts aus der politisch hoch fragilen Demokratischen Republik Kongo.

Während China seit langem eine ausgeprägte Rohstoffpolitik betreibt und sich beispielsweise in Afrika systematisch Rohstoffzugänge sichert, sind auf der europäischen und nationalen Ebene Politiken zur Sicherung der Rohstoffzugänge (noch) nicht erkennbar. Nach dem deutschen Politikverständnis der Subsidiarität zwischen Wirtschaft und Staat ist die Wirtschaft für die Sicherung der Rohstoffversorgung zuständig (BDI 2007). Insbesondere die kleinen und mittleren Unternehmen sind mit dieser Aufgabe allerdings überfordert. Die Integration einer Rohstoffpolitik in die Entwicklungs-, Außen- und Wirtschaftspolitik, welche die Anstrengungen der Wirtschaft flankiert, verbleibt eine Aufgabe der Zukunft.

\section{Anmerkungen}

1) In diesen Zahlen ist die Rohstoffnachfrage aus Verwendungssegmenten außerhalb der analysierten Zukunftstechnologien nicht enthalten.

2) Es handelt sich um Richtwerte. Die Angaben in den Quellen schwanken stark.

3) Coltan ist ein Erz aus dem Tantal und Niob gewonnen wird. Es wurde während des Bürgerkriegs in der Demokratischen Republik Kongo unter unzumutbaren Arbeitsverhältnissen und mit erheblichen Umweltlasten abgebaut. Die Erlöse flossen dem Waffenhandel zu.

4) Unter „tailored blanks“ sind maßgeschneiderte geschweißte Blechplatinen zu verstehen, die in einem Fertigungsgang hergestellt werden. Bei diesen Platinen werden spezielle Blechdicken jeweils dort eingesetzt, wo sie im Bauteil am effiziente- 
sten zur Gewichtsreduktion und Steifigkeitsverbesserung beitragen.

\section{Literatur}

Angerer, G.; Erdmann, L.; Marscheider-Weidemann, F. et al., 2009: Rohstoffe für Zukunftstechnologien. Der Einfluss des branchenspezifischen Rohstoffbedarfs in rohstoffintensiven Zukunftstechnologien auf die zukünftige Rohstoffnachfrage. Stuttgart

BDI - Bundesverband der Deutschen Industrie e.V., 2007: Rohstoffsicherheit - Anforderungen an Industrie und Politik. 2. BDI-Rohstoffkongress am 20. März 2007 in Berlin

Frondel, M.; Angerer, G.; Buchholz, P. et al., 2007: Trends der Angebots- und Nachfragesituation bei mineralischen Rohstoffen. Rheinisch-Westfälisches Institut für Wirtschaftsforschung RWI, Fraunhofer-Institut für System- und Innovationsforschung ISI und Bundesanstalt für Geowissenschaften und Rohstoffe BGR. Essen

GDCh-Gesellschaft Deutscher Chemiker; Dechema - Gesellschaft für Chemische Technik und Biotechnologie; DGMK - Deutsche Wissenschaftliche Gesellschaf für Erdöl, Erdgas und Kohle; VCI - Verband der Chemischen Industrie, 2010: Rohstoffbasis im Wandel. Gemeinsames Positionspapier der Chemieverbände. Frankfurt a. M.

Reuscher, G.; Ploetz, C.; Grimm, V.; Zweck, A., 2008: Innovationen gegen Rohstoffknappheit. Düsseldorf: Zukünftige Technologien Consulting der VDI Technologiezentrum $\mathrm{GmbH}$

Rohn, H.; Lang-Kroetz, C.; Pastewski, N.; Lettenmeier, M., 2009: Identifikation von Technologien, Produkten und Strategien mit hohem Ressourceneffizienzpotential. Projekt „Materialeffizienz und Ressourcenschonung“" (MaRess). Wuppertal

Statistisches Bundesamt, 2007: Statistisches Jahrbuch 2007. Für die Bundesrepublik Deutschland. Wiesbaden Statistisches Bundesamt, 2007: Volkswirtschaftliche Gesamtrechnung. Inlandsproduktberechnung. Lange Reihen ab 1970. Fachserie 18 Reihe 1.5. Wiesbaden

Statistisches Bundesamt, versch. Jahrgänge: Produzierendes Gewerbe. Kostenstruktur der Unternehmen des Verarbeitenden Gewerbes sowie des Bergbaus und Gewinnung von Steinen und Erden. Fachserie 4 Reihe 4.3. Wiesbaden, verschiedene Jahrgänge

Teipel, U. (Hg.), 2010: Rohstoffeffizienz und Rohstoffinnovationen. Stuttgart

USGS - United States Geological Survey, 2008: Mineral Commodity Summaries. Reston, VA, USA

$v b w$ - Vereinigung der Bayerischen Wirtschaft e.V., 2009: Rohstoffsituation in Bayern. Keine Zukunft ohne Rohstoffe. Strategien und Handlungsoptionen. Bericht der IW Consult GmbH Köln

Ziemann, S., 2009: Substitution knapper Metalle. Vertiefungsstudie zum BMBF-Projekt „Roadmap Umwelttechnologien 2020“. Karlsruhe: Institut für Technikfolgenabschätzung und Systemanalyse am Karlsruher Institut für Technologie

\section{Kontakt}

Dr. Gerhard Angerer

Hinterm Zaun 8, 76228 Karlsruhe

Tel: +49 (0) $160 / 8031046$

E-Mail: gerhard.angerer.ag@t-online.de 